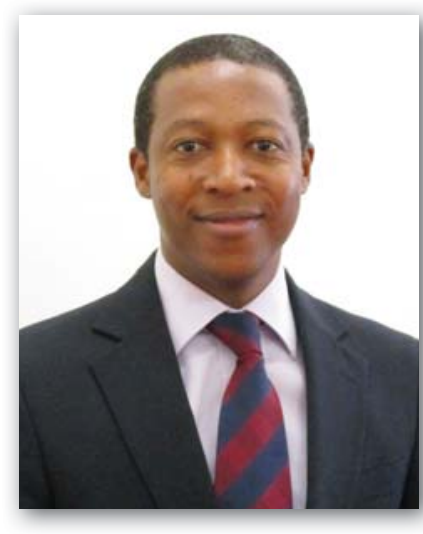

Editor, Ntobeko A.B. Ntusi

Professor of Medicine; Chair and Head, Department of Medicine,

University of Cape Town and Groote Schuur Hospital,

Observatory, South Africa

\title{
Cardiology in times of uncertainty
}

"Medicine is a science of uncertainty and an art of probability." William Osler (1849 - 19 19)

The words of Sir William Osler, echoed over a hundred years ago, ring as true today. Indeed, cardiologists are accustomed to clinical uncertainty in our practice, and a heuristic, probabilistic approach, aided by special investigations, is often what allows us to arrive at a likely diagnosis. We are comfortable to co-exist with and accept small degrees of uncertainty in clinical practice. Uncertainty is difficult to define, but refers to states of incomplete knowledge, where information may be lacking, ambiguous, conflicting, or untrustworthy. "Irrespective of its origin, we know uncertainty matters because when suppressed and ignored, consciously and subconsciously, it has potential to negatively impact on patients, their family, at a profoundly emotional level. It also affects health professionals, and it has been argued their tolerance of uncertainty can affect levels of investigation, patient safety, as well as healthcare resource use."(1-3)

Coronavirus disease 2019 (COVID-19) has spread at unprecedented speed and scale into a global pandemic with cardiovascular risk factors, phenotypes and complications emerging as important disease modifiers. Besides the untold global morbidity and mortality related to COVID-19, there has also been substantial uncertainty introduced in the practice of cardiology in the last year. Daily, whether cardiologists have been trying to define new clinical phenotypes, making diagnoses, deciding on appropriate investigational and therapeutic procedures, observing outcomes, the level of uncertainty that pervades our clinical practice has been unprecedented. In addition, there has been uncertainty related to how we communicate with patients and their families, how we assess our level of risk, and how we encounter system-level gaps in preparedness to deal with simple or complex presentations of the cardiovascular patient with COVID- 19. As health systems grapple with caring for patients affected with COVID-19, clinic visits and cardiovascular procedures that are deemed "elective" have been postponed, impacting significantly on the well-being of patients with chronic cardiovascular disorders. Uncertainty related to COVID- 19 extends beyond our professional lives: there is significant uncertainty about when we will have a third wave, what the magnitude of that surge will look like, the impact on family life and the activities we enjoy, our ability to travel and the economic impact on us, our loved ones and the national fiscus. 
Prolonged practice associated with uncertainty leads to mental ill-health. ${ }^{(4)}$ Health professionals who are uncertain about how a patient's condition may progress or respond to treatment may feel they have let patients and families down, particularly in the face of high mortality and absence of family members in care settings. (I) Moreover, some might make clinical decisions they feel uncomfortable about or disagree with, consequently leading to "moral injury" that may manifest as burn-out, stress, depression, anxiety, and post-traumatic stress disorder. . $^{(1,5)}$ Uncertainty may extend beyond specific patient management, particularly as many healthcare practitioners have found themselves working in new hospital environments where the stability and familiarity of a known clinical team are absent.

Djubelgovic, et al. argue that there is a finite amount of uncertainty in any clinical setting; and that there are several typologies of uncertainty in clinical medicine that are interlinked in a complex fashion (Figure I). ${ }^{(6)}$ In any clinical encounter, our knowledge rests somewhere between complete ignorance and absolute certainty. What most of us try to do daily is to increase the present state of knowledge about an index patient so that we minimise the quantum of reducible uncertainty. COVID- 19 has been associated with a reduction in the present state of knowledge, a reduction in the perfect state of knowledge, as well as an increase in the reducible uncertainty (red arrows). Epistemic uncertainty is intimately linked to the relationship between theory, evidence, and knowledge and cannot be reduced. The relationships among observed, observable, and unobservable realities express uncertainties that can be characterised as a lack of knowledge about what is known (unknown knowns), what is known to be unknown (known unknowns), and not knowing what is unknown (unknown unknowns). Intricately linked with this classification of uncertainty is the psychological taxonomy that categorises uncertainty based on knowledge of the external world and on our own state of knowledge. It is suggested that any attempt to develop a comprehensive treatise of uncertainty in clinical medicine must consider the insights obtained from psychological research on uncertainty. Uncertainty can be effectively managed by explicitly recognising its many sources, improving the quality of medical evidence, using better information technology tools, searching for sources of bias, and applying probability and decision theory to decisions under uncertainty. ${ }^{(6)}$ 


\section{Editor,}

Ntobeko A.B. Ntusi

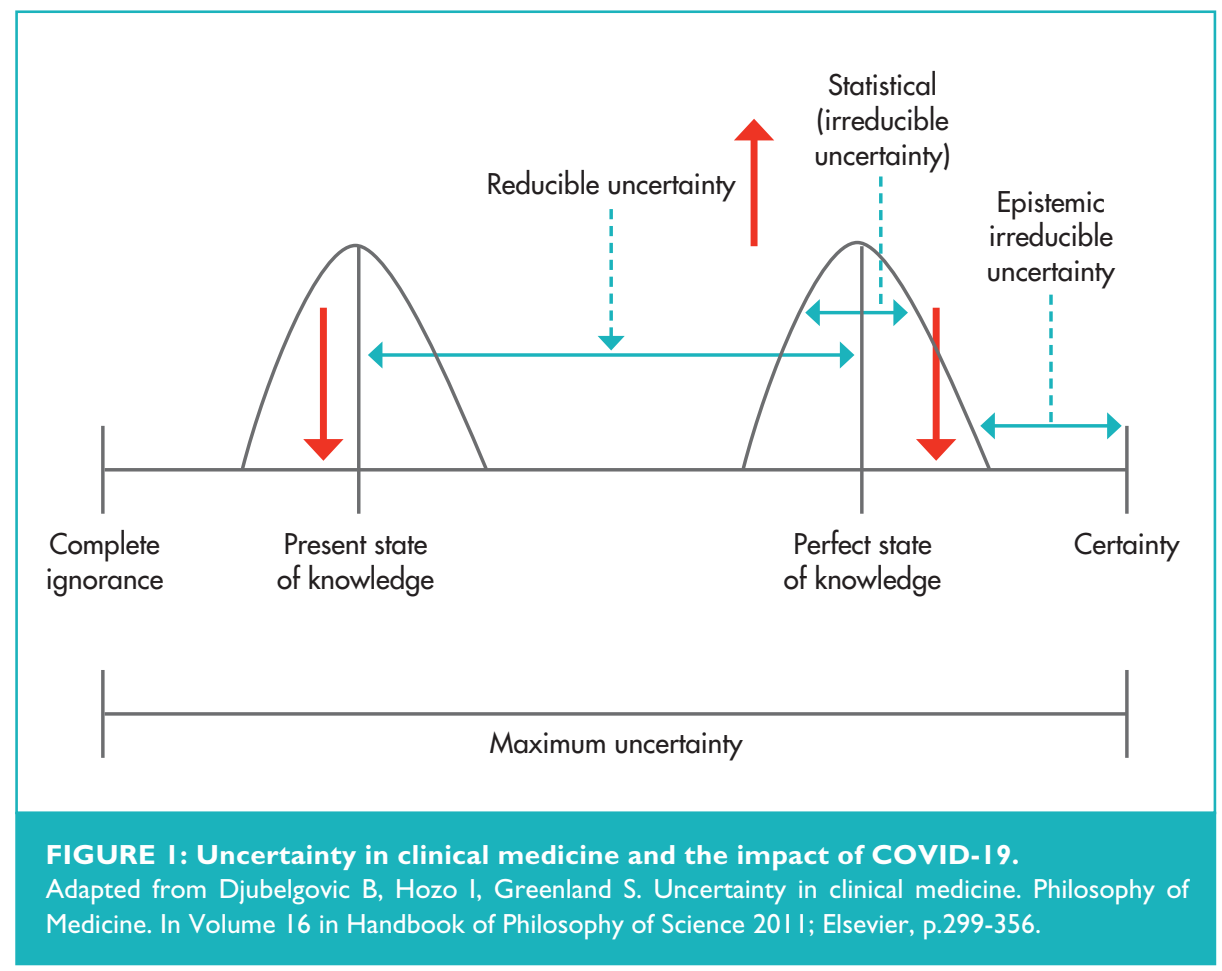

How should we be approaching uncertainty as cardiologists in the time of COVID-19? In times of crisis, our natural inclination is to forge ahead in search of answers, recognising our call to duty and the need for rapid decision-making and execution. In these uncertain times, we need to build our individual and collective resilience by constantly focusing on our development within each of the 6 domains below: (i) vision, clarity, and congruence; (ii) composure; (iii) reasoning; (iv) tenacity; (v) collaboration; and (vi) personal and organisational health. For instance, in the first domain, important questions to ask may be: (a) what is my vision to get through this time? (b) what is of critical importance to support my vision? (c) what can be placed on the backburner for a while? By considering simple questions within each domain, and seeking deliberate responses, you will slow your mind down and regain a sense of control. Thus, allowing the opportunity to reboot and refocus on how to best navigate your well-being during times of crisis and uncertainty. ${ }^{(7)}$ Such an approach will lead us to navigate unchartered waters with a greater sense of control, support for others, and a clear vision to tackle the days, weeks, months, and years ahead.

While unsettling, we need to embrace uncertainty, as it is often what drives evolution in cardiology. Uncertainty about the application of existing evidence to the individual patient in front of us has yielded personalised, algorithm-based precision medicine, which is increasingly available and will undoubtedly impact significantly on how cardiovascular care is delivered, not only for COVID-19 patients. COVID-19 has accelerated healthcare reform efforts forcing 
cardiologists to rethink the way care is delivered. Similarly, cardiovascular education has relied substantially on technology to enable increased access and remote learning. These online platforms and mobile applications have enabled shared decision-making among clinicians and with patients. The uncertainty of the current crisis may present opportunities to improve the practice and education of cardiology in the future.

The uncertainty related to COVID- 19 has also impacted on the Journal. The SA Heart ${ }^{\circledR}$ congress for 2020 was cancelled and a congress issue was not to be. As we were finalising a 4th issue of the Journal in 2020, the second wave engulfed us, and attention swiftly moved towards clinical efforts. There has also been a notable delay in the return of peer reviews in the past year, something we hope to change as this is a fundamental aspect of our work.

In this issue of the Journal, we have obituaries for 2 colleagues who passed away in 2020: Professor Lungile Pepeta and Professor Solly Levin. As you read these, I hope that you will pause to also remember the many colleagues and friends we have lost in the past year to COVID-19.

\section{Conflict of interest: none declared.}

\section{REFERENCES}

I. Koffmann JK, Gross J, Etkind SN, Seman L. Uncertainty and COVID-19: how are we to respond? J Royal Soc Med 2020;1 I 3(6):21 I 216. https://doi.org//0.1 177/0141076820930665

2. Schwarze ML, Taylor LJ. Managing uncertainty - Harnessing the power of scenario planning. N Engl J Med 20 I7;377(3):206-208. https://www.nejm.org/doi//0.1056/NEJMp I704|49

3. Simpkin AL, Schwartzstein RM. Tolerating uncertainty - the next medical revolution? N Engl J Med 2016;375(I8):17/3-1715. https://www.nejm.org/doi// 0.1056/NEJMp I 606402

4. Bana T, Hoare J, Letuka P, Ntusi N. COVID-19 and impact of psychological stress on cardiovascular disease. S Afr Heart J 2020;17(3):282-286. https://www.journals.ac.za/index.php/SAHJ/article/view/4374

5. Cook D, Rocker G. Dying with dignity in the intensive care unit. N Engl J Med 20 | 4:370(26):2506-25 |4. https://www.nejm.org/doi/full// 0.1 056/nejmral 208795

6. Djubelgovic B, Hozo I, Greenland S. Uncertainty in clinical medicine. Philosophy of Medicine. In Volume 16 in Handbook of Philosophy of Science 20 I I; Elsevier, p.299-356. ISBN: 97804445 I 7876. https://doi.org/ I 0. I 0 I 6/B978-0-444-5 I 787-6.500 I I-8

7. Rossouw JG, Erieau CL, Beeson ET. Building resilience through a virtual coach called Driven: Longitudinal pilot study and the neuroscience of small, frequent learning tasks. Int J Neuropsychother 2019;7(2):23-4I.

https://doi.org/| 0.12744/ijnpt.2019.023-04| 